\title{
A supplementary study of response uncertainty and relative expected value in multiple-choice decision behavior
}

\author{
DAVIO M. MESSICK, UNIVERSITY OF CALIFORNIA, SANTA BARBARA \\ AMNON RAPOPORT, UNIVERSITY OF NORTH CAROLINA, CHAPEL HILL
}

Data from a previous study are reanalyzed. The study is typical 10-choice probability learning task including 1500 trials. The results demonstrate the feasibility of decomposing multiple-choice decision behavior into two distinct processes. The first process is concerned with the uncertainty of the response distribution, and the second process is concerned with the allocation of the responses to the available alternatives

In a previous study investigating human decision strategies in a multiple-choice decision task, Messick \& Rapoport (1965a) analyzed Ss' behavior in terms of the expected value, $V$, and the uncertainty, $H$, of the individual response distributions. These statistics are defined as follows for a block of $m$ trials in which $\mathrm{S}$ may make any of $\mathrm{n}$ alternative responses on each trial:

$$
\text { n }
$$

and

$$
\text { (2) } H=-\sum_{i=1}^{n}[f(i) / m] \log _{2}(f(i) / m) \text {. }
$$

In these expressions, $f(i)$ is the frequency of response with alternative $i$, and $E_{i}(g)$ is the expected gain for one response with the ith alternative. $V$ is interpreted as a measure of the efficiency of S's behavior within a block of $\mathrm{m}$ trials, and $\mathrm{H}$ may be taken as a measure of the predictability of S's behavior.

In a subsequent paper (Messick \& Rapoport, 1965b), multiple-choice decision behavior was conceptualized as being composed of two distinct processes, the first involving the determination of $\mathrm{H}$ and the second having to do with the allocation of responses to different alternatives. A measure of the efficiency of this latter process may be obtained by computing the maximum and minimum values of $V$ for a given value of $H$. The details of these computations are described by Messick \& Rapoport (1965b). A measure of the efficiency of the allocation process, which is independent of $\mathrm{H}$, may be obtained by the ratio

\section{(3)

$$
R=\left(V^{\prime}-V_{\min }\right) /\left(V_{\max }-V_{\min }\right),
$$

where $V^{\prime}$ is some observed value of $V$. The range of $R$ is the unit interval. The closer $R$ to unity, the closer $S$ is to maximizing his expected gain given the uncertainty of his response distribution.

The above-mentioned study investigated $R$ as a
Table 1. Stimulus distributions for groups IV, V, and VI

\begin{tabular}{llll} 
Event & IV & $\begin{array}{c}\text { Group } \\
V\end{array}$ & VI \\
\hline 0 & .01 & .03 & .05 \\
1 & .03 & .06 & .10 \\
2 & .06 & .13 & .17 \\
3 & .10 & .10 & .10 \\
4 & .12 & .09 & .05 \\
5 & .19 & .13 & .07 \\
6 & .28 & .27 & .29 \\
7 & .14 & .12 & .10 \\
8 & .06 & .05 & .05 \\
9 & .01 & .02 & .02 \\
\hline
\end{tabular}

function of $\mathrm{H}$ in a task in which $\mathrm{H}$ was experimentally fixed. It was found that while $R$ increased as a function of trial blocks, there was no relationship between $R$ and $H$. In the present report data from a previously published experiment are analyzed with respect to the conceptualization offered by Messick \& Rapoport (1965b). The study is a 10-choice probability learning task in which the predictability, $\mathrm{H}$, of S's responses is not experimentally controlled.

\section{Method}

The details of this experiment may be found in Beach \& Shoenherger (1965). ${ }^{1}$ Only a brief description of the procedure will be presented here.

The Ss were newly comissioned Naval officers, who were divided into three groups (Groups IV, V, and VI of Beach \& Shoenberger, the only groups for which data were available) of 15, 13, and 13 Ss respectively. The task of $S$ was to predict which of 10 white event lights, labelled 0 to 9 , would occur on each trial. Ss were run for $1 \mathrm{hr}$. a day until they reached a total of 1500 trials. No monetary reward was given to Ss. The stimulus distributions were different for the three different groups. These stimulus distributions are presented in Table 1.

\section{Results}

The results of our analysis of the data from this study are presented in terms of blocks of 100 trials.

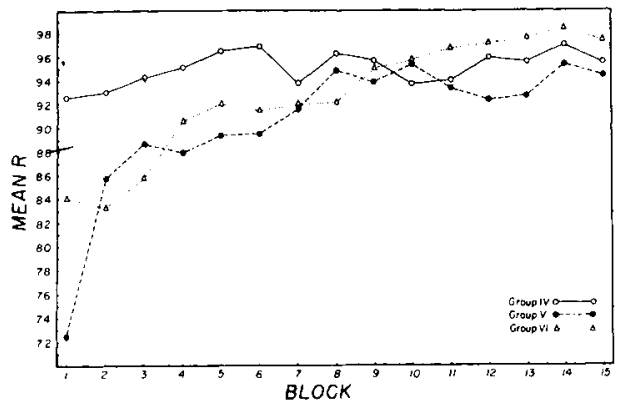

Fig. 1. Mean $\mathbf{R}$ for groups IV, $\mathrm{V}$, and VI. 


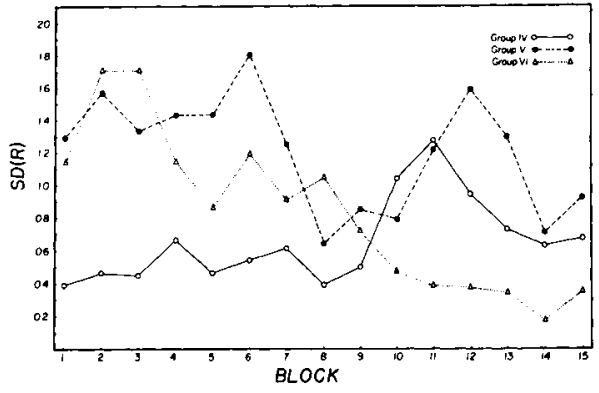

Fig. 2. Standard deviations of $\underline{\mathbf{R}}$ for groups IV, $\mathbf{V}$, and VI.

For each block the $\mathrm{R}$ and $\mathrm{H}$ statistics were computed for each $S$ in each of the three groups. The mean $R$ is presented in Fig. 1 as a function of trial blocks. It can be seen that the mean $R$ increases, approaching an asymptote which is close to unity. The SD of the $\mathbf{R}$ measures in the three groups is shown in Fig. 2 as a function of trial blocks. While the SD tends to be somewhat erratic, there is not evidence, with the possible exception of Group IV, that it is increasing.

Figure 3 displays the mean $\mathrm{H}$ as a function of trial blocks. Not only does $\mathrm{H}$ decrease as a function of time, in addition there is no evidence that this statistic has reached an asymptote. The form of the function appears approximately linear. While the mean $H$ decreases, the $\mathrm{SD}$ of the $\mathrm{H}$ measure shows an increase as a function of trial blocks as can be seen in Fig. 4 .

Discussion

These results further demonstrate the feasibility of decomposing multiple-choice decision behavior into two distinct processes. The first of these processes is concerned with the allocation of the responses to the available alternatives. It has been shown that the efficiency of this process as measured by $R$, increases continuously, approaching an asymptote which is close to unity. Thus, taking the uncertainty of S's response distribution into account, the behavior becomes nearly optimal.

However, most Ss do not maximize expected gain in

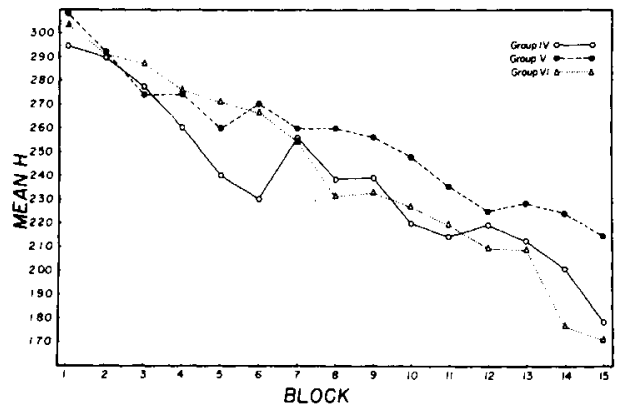

Fig. 3. Mean $\mathbf{H}$ for groups IV, $\mathrm{V}$, and $\mathrm{VI}$.

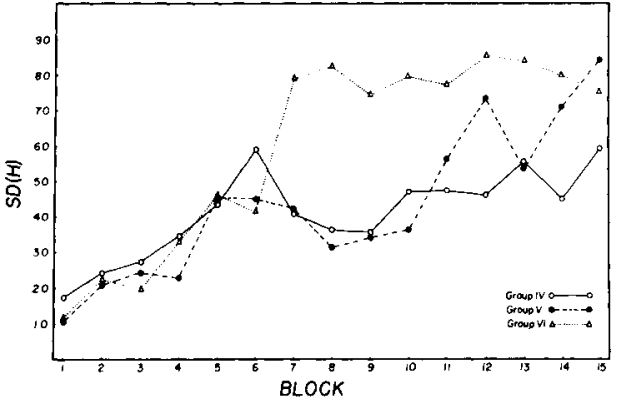

Fig. 4. Standard deviations of $\underline{\mathrm{H}}$ for groups IV, V, and VI.

a strict sense since the response uncertainty does not go to zero. Thus, the problem of the discrepancy between actual and optimal behavior is reduced to the problem of accounting for $\mathbf{H}$.

The results also indicate that, contrary to our expectations, $\mathrm{H}$ decreases over 1500 trials without reaching an asymptote. This finding portends difficulties for theories of choice which imply that behavior in a multiple-choice task will reach a steady state after a relatively small number of trials. With regard to the uncertainty of S's behavior as measured by $\mathrm{H}$, we find no evidence that this occurs in 1500 trials.

The data reported here tend to question even more seriously the assumption that the underlying choice process or learning process is homogeneous across Ss. It was shown above that the differences between $\mathrm{Ss}$ in $\mathrm{H}$ tend to increase as a function of trial blocks as reflected in the increasing SD of this measure. This fact would seem to imply that the distributions of choices for Ss do not approach a common asymptote as would be expected from the models of Estes (1959) or Siegel (1959)..$^{2}$

\section{References}

Beach, L. R., \& Shoenberger, R. W. Event salience and response frequency in a ten-alternative probability learning situation. $J$. exp. Psychol., 1965, 69, 312-316.

Estes, W. K. The statistical approach to learning theory. In $\mathbf{S}$. Koch (Ed.), Psychology: a study of a science. Vol. 2. New York: McGraw-Hill, 1959, 380-491.

Messick, D. M., \& Rapoport, A. A comparison of two payoff functions on multiple-choice decision behavior. J. exp. Psychol., $1965 \mathrm{a}, 69,75-83$.

Messick, D. M., \& Rapoport, A. Expected value and response uncertainty in multiple-choice decision behavior. $J$, exp. Psychol, $1965 \mathrm{~b}, 70,224-230$.

Siegel, S. Theoretical models of choice and strategy behavior: stable state behavior in the two-choice uncertain outcome situation. Psychometrika, 1959, 24, 303-316.

\section{Notes}

1. The authors want to thank L. R. Beach \& R. W. Shoenberger for making their data available.

2. Essentially the same results were obtained from a reanalysis of the 10-choice probability learning study reported by Messick \& Rapoport (1965a). 